University of Nebraska - Lincoln

DigitalCommons@University of Nebraska - Lincoln

January 2008

Experimental evidence of strain relaxed domain structure in (100)/(001)-oriented epitaxial lead titanate thick films grown by metal organic chemical vapor deposition

Hiroshi Nakaki

Tokyo Institute of Technology, Yokohama, Japan

Yong Kwan Kim

Tokyo Institute of Technology, Yokohama, Japan

Shintaro Yokoyama

Tokyo Institute of Technology, Yokohama, Japan

Rikyu Ikariyama

Tokyo Institute of Technology, Yokohama, Japan

Hiroshi Funakubo

Tokyo Institute of Technology, Yokohama, Japan

See next page for additional authors

Follow this and additional works at: https://digitalcommons.unl.edu/physicsgruverman

Part of the Physics Commons

Nakaki, Hiroshi; Kim, Yong Kwan; Yokoyama, Shintaro; Ikariyama, Rikyu; Funakubo, Hiroshi; Streiffer, S. K.; Nishida, Ken; Saito, Keisuke; and Gruverman, Alexei, "Experimental evidence of strain relaxed domain structure in (100)/(001)-oriented epitaxial lead titanate thick films grown by metal organic chemical vapor deposition" (2008). Alexei Gruverman Publications. 44.

https://digitalcommons.unl.edu/physicsgruverman/44

This Article is brought to you for free and open access by the Research Papers in Physics and Astronomy at DigitalCommons@University of Nebraska - Lincoln. It has been accepted for inclusion in Alexei Gruverman Publications by an authorized administrator of DigitalCommons@University of Nebraska - Lincoln. 


\section{Authors}

Hiroshi Nakaki, Yong Kwan Kim, Shintaro Yokoyama, Rikyu Ikariyama, Hiroshi Funakubo, S. K. Streiffer, Ken Nishida, Keisuke Saito, and Alexei Gruverman 


\title{
Experimental evidence of strain relaxed domain structure in (100)/(001)-oriented epitaxial lead titanate thick films grown by metal organic chemical vapor deposition
}

\author{
Hiroshi Nakaki, ${ }^{1}$ Yong Kwan Kim, ${ }^{1}$ Shintaro Yokoyama, ${ }^{1}$ Rikyu Ikariyama, ${ }^{1}$ \\ Hiroshi Funakubo, ${ }^{1}$ S. K. Streiffer, ${ }^{2}$ Ken Nishida, ${ }^{3}$ Keisuke Saito, ${ }^{4}$ and Alexei Gruverman ${ }^{5}$ \\ ${ }^{1}$ Department of Innovative and Engineered Material, Tokyo Institute of Technology, Yokohama \\ 226-8503, Japan \\ ${ }^{2}$ Center for Nanoscale Materials, Argonne National Laboratory, Argonne, Illinois 60439, USA \\ ${ }^{3}$ Department of Electric Engineering, National Defense Academy, Yokohama 239, Japan \\ ${ }^{4}$ Application Laboratory, Bruker AXS, 3-9-A Moriya-cho, Kanagawa-ku, Yokohama 221-0022, Japan \\ ${ }^{5}$ Department of Physics and Astronomy, University of Nebraska-Lincoln, Lincoln, Nebraska 68588, USA
}

(Received 20 February 2008; accepted 27 July 2008; published online 26 September 2008)

Epitaxial (100)/(001)-oriented $\mathrm{PbTiO}_{3}$ films with thickness of $2.8 \mu \mathrm{m}$ were grown on Nb-doped (100) $\mathrm{SrTiO}_{3}$ substrates by pulsed metal organic chemical vapor deposition. Complex domain structures consisting of $c$-domains (c1) and three types of $a$-domains (a1, a2, and a3) were observed by piezoresponse force microscopy in conjunction with high-resolution x-ray diffraction analysis. The obtained results suggest that (a2/a3) and (a1/c1) domain configurations represent mostly strain-relaxed structures. This conclusion is in good agreement with theoretical predictions made for thick films [Phys. State., Solidi 37, 329 (1976)]. (C) 2008 American Institute of Physics.

[DOI: 10.1063/1.2981193]

Ferroelectric films have been extensively studied for various applications including nonvolatile ferroelectric random access memory devices and microelectromechanical systems (MEMSs). ${ }^{1}$ Understanding the contribution of ferroelectric domains to ferroelectric and piezoelectric characteristics is essential to the development of ferroelectric films with optimized properties.

It is well known that the formation of domain structure in ferroelectric films is affected by the mechanical strains arising from lattice mismatch and/or thermal expansion cofficient difference between the film and substrate. ${ }^{2-4}$ In ferroelectric films with tetragonal perovskite structure, such as $\mathrm{PbTiO}_{3}$ or $\mathrm{Pb}(\mathrm{Zr}, \mathrm{Ti}) \mathrm{O}_{3}(\mathrm{PZT})$, there are three possible domain types: $c$-domains with their polarization direction along the surface normal and two types of $a$-domains (defined as $a$ and $a^{\prime}$ ) with their polarization axes rotated by approximately $90^{\circ}$ and oriented in the plane of the substrate. In such films, the phase transition from the cubic to tetragonal phase is accompanied by the formation of domain configurations consisting of $a / a^{\prime}$ or $a / c$ or $a^{\prime} / c$ domains (90 domains) due to strain relaxation during the cooling process. Theoretical treatment of strain relaxation via $90^{\circ}$ domain formation as well as film thickness effects on domain configuration has been done by Roytburd ${ }^{5}$ and Roytburd and Yu. ${ }^{6}$ However, there have been very few reports on experimental studies of domain structures in epitaxial thick films, especially above $1 \mu \mathrm{m}$ in thickness, even though this film thickness regime is important for MEMS applications. Recently, a novel domain structure, labeled as type III, was experimentally discovered in (100)/(001)-oriented epitaxial $\mathrm{PbTiO}_{3}$ thick films grown on (100) $\mathrm{SrTiO}_{3}$ substrates by metal organic chemical vapor deposition (MOCVD). ${ }^{7}$ This is different from the well defined one labeled as type II observed for the films below $1 \mu \mathrm{m}$ in thickness. In this paper, we report the details of domain arrangement in thick epitaxial $\mathrm{PbTiO}_{3}$ films with thickness of $2.8 \mu \mathrm{m}$ as investigated by means of piezoresponse force microscopy (PFM) and high-resolution x-ray diffraction (XRD). PFM is widely used to visualize the threedimensional domain configurations in ferroelectrics, ${ }^{8,9}$ while $\mathrm{XRD}$ is useful for detailed analysis of the crystal structure of constituent domains. ${ }^{10,11}$

$\mathrm{PbTiO}_{3}$ films with thickness of $2.8 \mu \mathrm{m}$ were grown at $600{ }^{\circ} \mathrm{C}$ on $\mathrm{Nb}$-doped (100) $\mathrm{SrTiO}_{3}$ substrates by pulsed MOCVD. Vapor of $\mathrm{Pb}\left(\mathrm{C}_{11} \mathrm{H}_{19} \mathrm{O}_{2}\right)_{2}$ and $\mathrm{Ti}\left(\mathrm{O} \cdot i-\mathrm{C}_{3} \mathrm{H}_{7}\right)_{4}$ was used as $\mathrm{Pb}$ and $\mathrm{Ti}$ source materials, respectively, and oxygen gas was used as an oxidant. The source gas mixture was alternatively supplied to the substrate in a cold-wall-type vertical reaction chamber. Details of the film preparation are described elsewhere. ${ }^{12,13}$

Using a calibrated $\mathrm{x}$-ray fluorescence spectrometer (PANalytical PW-2404), the Pb/Ti ratio of the films used in the present study was determined to be almost 1.0. Crystal structure of the films was characterized by high-resolution XRD (PANalytical X'Pert MRD). The surface normal direction of the $\mathrm{SrTiO}_{3}$ substrates was defined as $\mathrm{SrTiO}_{3}$ [001]. The XRD $\omega-2 \theta$ scans indicated that the films consisted of a single perovskite $\mathrm{PbTiO}_{3}$ phase and were epitaxially grown with the surface normally oriented to $(100) /(001)$. Analysis of domain structure configuration was carried out by PFM using vertical (out-of-plane) and lateral (in-plane) imaging modes. $^{14}$

Figure 1(a) shows the amplitude vertical PFM image of the $\mathrm{PbTiO}_{3}$ film, which consists of two types of regions labeled as region I and region II. Bright contrast in region I corresponds to the "up" state of the polarization along the surface normal while black contrast in the region II stripes oriented in the [100] $\mathrm{SrTiO}_{3}$ direction is indicative of inplane polarization. 

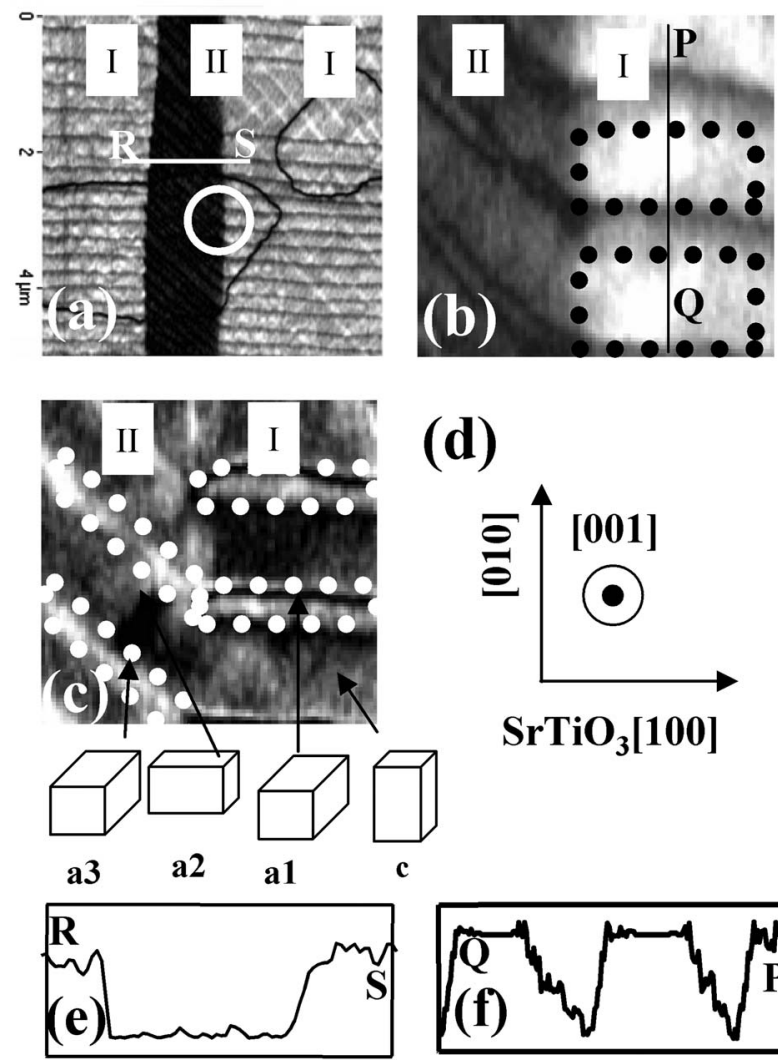

(d)

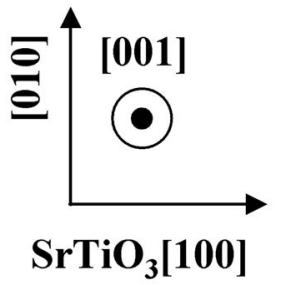

FIG. 1. (a) Vertical PFM amplitude image of the $\mathrm{PbTiO}_{3}$ film. (b) Magnified vertical PFM amplitude image of the area marked by the white circle in (a). (c) Lateral PFM amplitude image of the same area as in (b). (d) Sketch of crystal orientation of the $\mathrm{SrTiO}_{3}$ substrate. (e) Cross-section profile of the vertical PFM signal across the line $R-S$ in (a). (e) Cross-section profile of the vertical PFM signal along the line $P-Q$ in (b).

Figures 1(b) and 1(c) show magnified vertical and lateral PFM amplitude images, respectively, for the region marked by the white circle in Fig. 1(a). Analysis of these images revealed that region I consisted of $c$-domains and $a$-domains with polar axis along the [010] $\mathrm{SrTiO}_{3}$ direction-a configuration termed (c/a1). On the other hand, it was concluded that gray/bright areas in region II correspond to (a2/a3) domain configuration composed of two types of $a$-domains with the polarization axis along the [100] $\mathrm{SrTiO}_{3}$ and [010] $\mathrm{SrTiO}_{3}$ directions (a2 and a3 domains, respectively) with the domain boundary between $\mathrm{a} 2$ and $\mathrm{a} 3$ domains being oriented along ( $\left(\begin{array}{lll}1 & \overline{1} & 0\end{array}\right)$ plane as shown in Fig. 1(c).

It is important to note that there are three kinds of $a$-domains in Fig. 1 that have not been previously reported. There are two possible orientations of $90^{\circ}$ domain boundaries in region I: along $\left(\begin{array}{lll}0 & \overline{1} & 1\end{array}\right)$ and (011) planes. Figure 1(f) shows a line scan profile of the vertical PFM signal across line $P-Q$ in Fig. 1(b). The asymmetric vertical PFM signal inclination along this line near the domain boundary is attributed to the (011) plane of an (a1/c)-domain wall. Similarly, in Fig. 1(e) the (011) plane of an (a2/c)-domain boundary gives rise to the asymmetric shape of the $R-S$ line profile [Fig. 1(a)] near the domain boundary. Three-dimensional images of domain boundaries are schematically illustrated in Fig. 2 based on these results. (d)

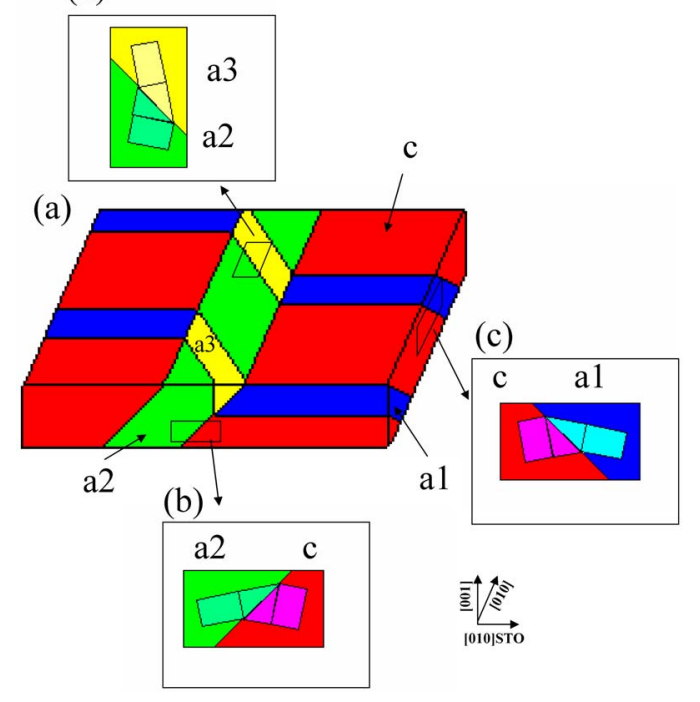

FIG. 2. (Color online) Schematic of (a) the three-dimensional domain structure shown in Fig. 1 and $[(b)-(d)]$ the different domain configurations based on the results shown in Fig. 1.

The same films were subsequently investigated by highresolution XRD. Figure 3(a) shows a $\omega-\psi$ map of the $\mathrm{PbTiO}_{3}$ 002 , corresponding to the $c$-axis of $\mathrm{PbTiO}_{3}$. Such a map samples diffraction in plane that is approximately the 001 zone axis and the second Laue zone with the center position $\left(\omega=\psi=0^{\circ}\right)$ the $\mathrm{SrTiO}_{3}$ [001], i.e., the surface normal orientation. This map contains peaks corresponding to $c$-domains in Fig. 1. Figure 3(a) shows four spots at $\omega=\psi=\left|0.7^{\circ}\right|$. This means that four $c$-domains with different orientations are formed in this film and are labeled from "C-1" to "C-4" as shown in Fig. 3(a).

Figure 3(b) shows a $\omega-\psi$ scan of the $\mathrm{PbTiO}_{3} 200$ along the $\mathrm{SrTiO}_{3}$ [001]. It must be noted that the center peak in Fig. 3(b) comes from the substrate $\mathrm{SrTiO}_{3}$ 200. Figure 3(b) indicates two kinds of spots: eight spots at $\omega \neq 0^{\circ}$ and $\psi$ $\neq 0^{\circ}$ labeled from "A-1" to "A- 8 " and four spots at $\omega=0^{\circ}$ or $\psi=0^{\circ}$ labeled from "B-1" to "B-4." The results shown in Figs. 3(a) and 3(b) are similar to the previously reported data. ${ }^{7}$ However, XRD observation of two types of $a$-domains is not consistent with the PFM data analysis indicating three types of $a$-domains. This inconsistency can be explained by the presence of the strain-free domain walls. It must be mentioned that the surface normal lattice constants along the $a$ and $c$-axes and $a_{\perp}$ and $c_{\perp}$ are found to be $a_{\perp}=0.3900 \mathrm{~nm}$ and $c_{\perp}=0.4148 \mathrm{~nm}$ in close agreement with the values reported for the strain-free powder, which suggests the existence of strain-free $90^{\circ}$ domain boundaries in the present $\mathrm{PbTiO}_{3}$ thick films.

Given that the $c$-domains are tilted from the surface normal as shown in Fig. 3(a), the $a$-axis of the $c$-domains should be also tilted from the in-plane direction provided that the unit cell keeps the tetragonal symmetry. To check the inplane direction, the in-plane $\omega-\psi$ map of $\mathrm{PbTiO}_{3} 200$ along $\mathrm{SrTiO}_{3}[100]$, which is expected to be paired with the "C-2" and "C-3" spots in Fig. 3(a), was measured [Fig. 3(c)]. In total, there are three spots: two spots at $\psi=87.9^{\circ}$ labeled as "D-1" and "D-2" and one spot at $\psi=89.3^{\circ}$ labeled as "E-1." 

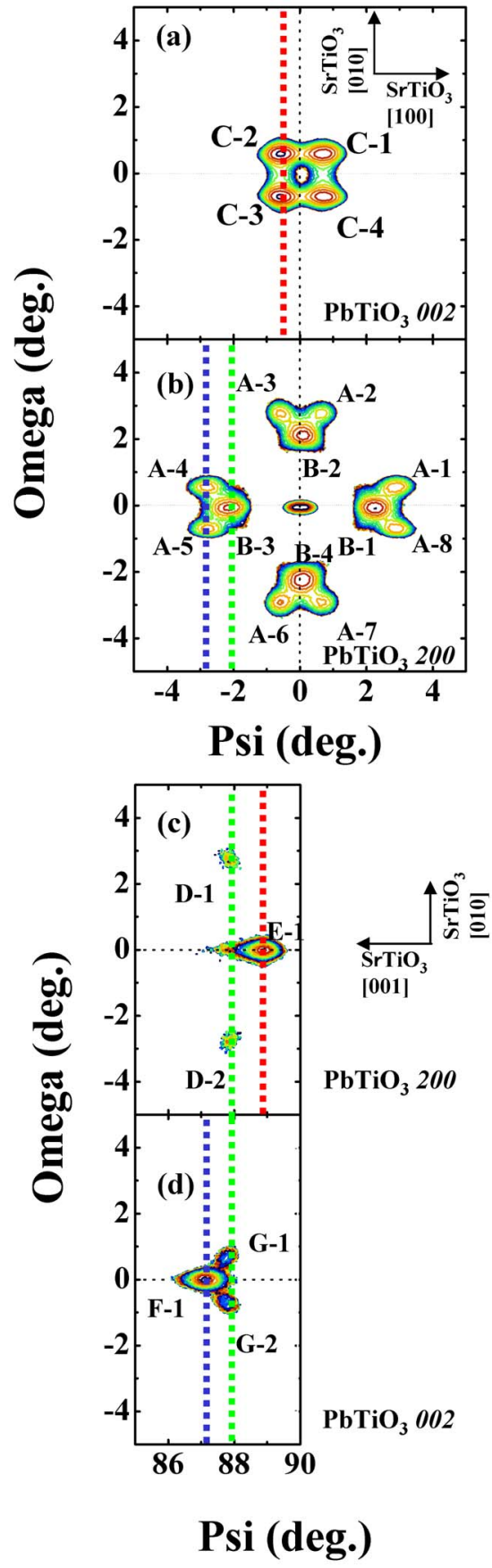

FIG. 3. (Color online) $\omega-\psi$ scans of XRD pattern (a) out-of-plane 002 and (b) out-of-plane 200. (c) In-plane 200 along $\mathrm{SrTiO}_{3}$ [100] and (d) in-plane 002 along $\mathrm{SrTiO}_{3}[100]$.

In the case of a tetragonal perovskite crystal such as $\mathrm{PbTiO}_{3}$, the internal angle of the unit cell should be equal to $90^{\circ}$. This means that spots paired with the $\mathrm{C}-2$ and $\mathrm{C}-3$ spots located at $\psi=-0.7^{\circ}$ in Fig. 3(a) should be at $\psi=89.3^{\circ}$ in Fig. 3(c). Therefore, the E-1 peak located at $\psi=89.3^{\circ}$ is a paired one and C-2-E-1 and/or C-3-E-1 are the combinations. The domains corresponding to the spot combinations of C-2-E-1 and C-3-E-1 are schematically shown in Figs. 4(a) and 4(b), respectively.

On the other hand, for $a$-domains in Fig. 3(b), there are two possible directions of the polar axis-along $\mathrm{SrTiO}_{3}$ [100] and $\mathrm{SrTiO}_{3}[010]$ — so that there is a possibility of

\section{c-domains}

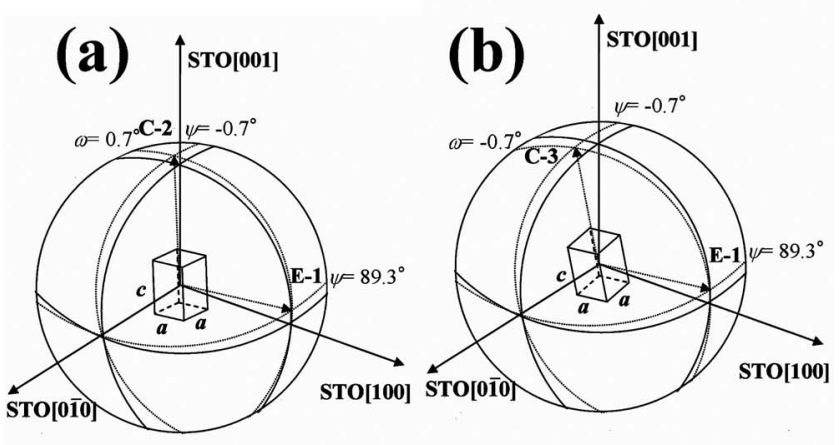

\section{a-domains}
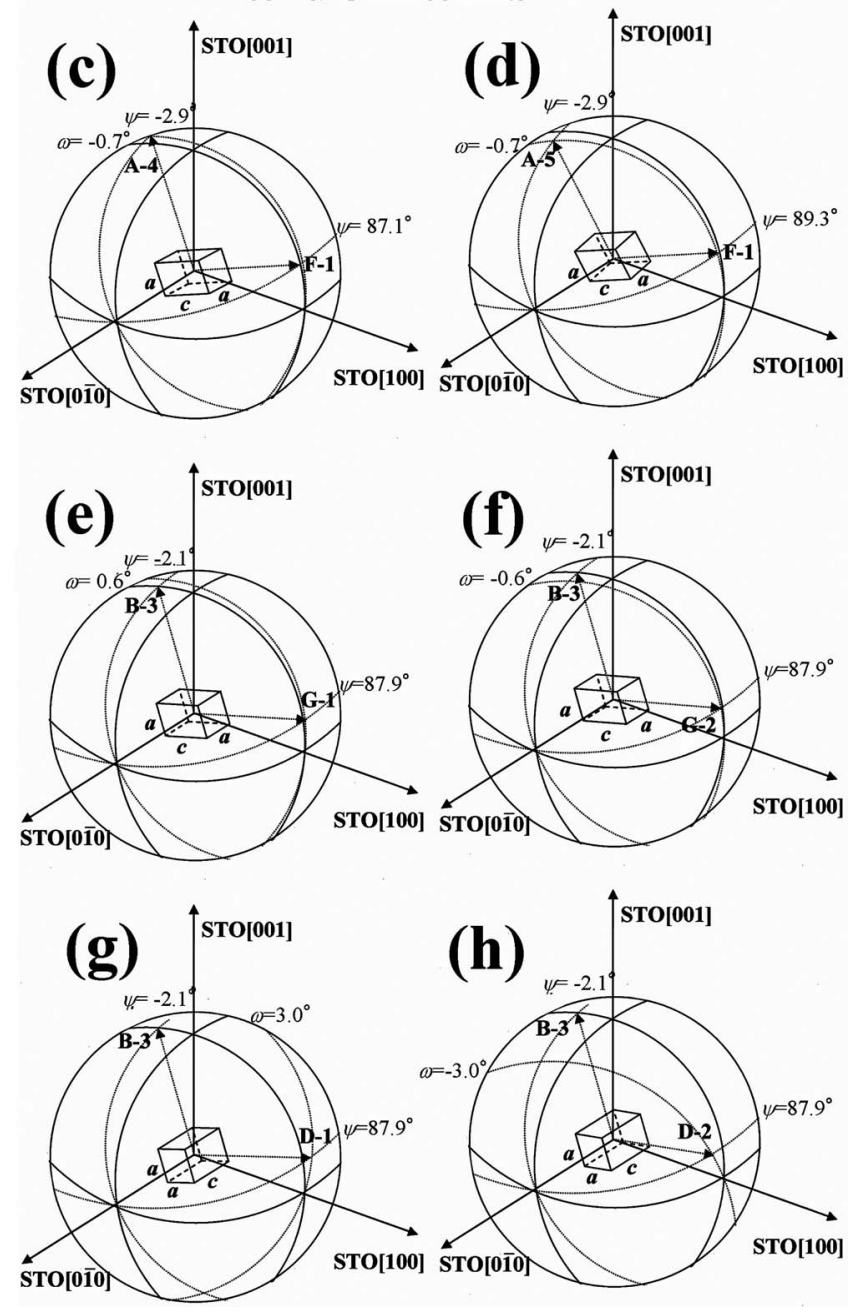

FIG. 4. Schematic of the domains obtained by the results shown in Fig. 3(a). C-2 spot and E-1 spot combination shown in Fig. 3(b). C-3 spot and E-1 spot combination shown in Fig. 3(c). A-4 spot and F-1 spot combination shown in Fig. 3(d) A-5 spot and F-1 spot combination shown in Fig. 3. (e) B-3 spot and G-1 spot combination shown in Fig. 3(f). B-3 spot and G-2 spot combination shown in Fig. 3(g). B-3 spot and D-1 spot combination shown in Fig. 3(h). B-3 spot and D-2 spot combination shown in Fig. 3.

$a$-axis and $c$-axis along the in-plane $\mathrm{SrTiO}_{3}[100]$ direction. The in-plane $\omega-\psi$ maps of $\mathrm{PbTiO}_{3} 200$ and $\mathrm{PbTiO}_{3} 002$ measured along $\mathrm{SrTiO}_{3}[001]$ are shown in Figs. 3(c) and 3(d), respectively. For an internal angle of $90^{\circ}$ (as in normal tetragonal $\mathrm{PbTiO}_{3}$ ), the corresponding spots for "A-4" and "A-5" located at $\psi=-2.9^{\circ}$ in Fig. 3(b) must be located at 


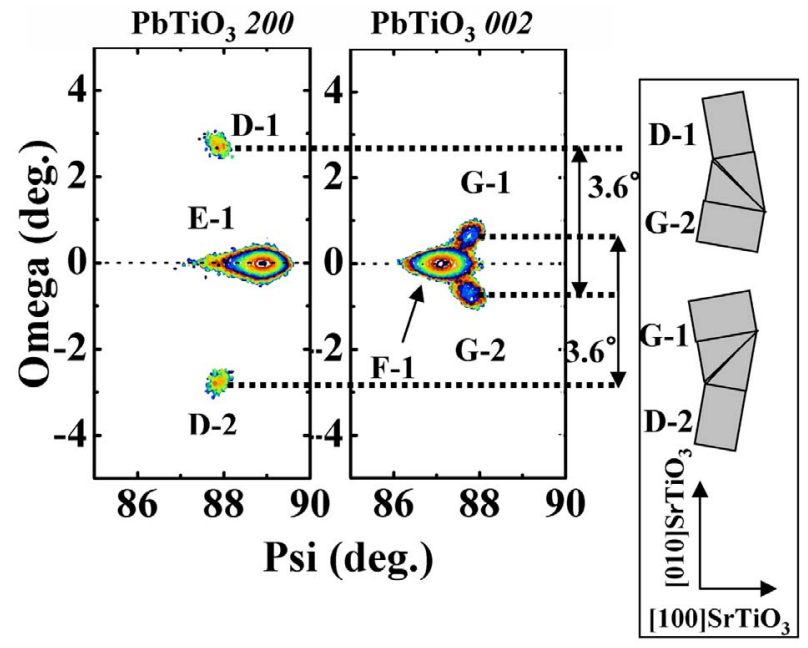

FIG. 5. (Color online) $\omega-\psi$ scans of XRD patterns of (a) out-of-plane 200, (b) in-plane 200 along $\mathrm{SrTiO}_{3}$ [100], and (c) schematic of the possible domain combination.

$\psi=87.1^{\circ}$ in Fig. 4(c) and/or Fig. 4(d). "F-1" spots in Fig. $3(d)$ are located at $\psi=87.1^{\circ}$, which will be overlapped spots corresponding to A-4 and A-5 along the surface normal direction. Therefore, domains which correspond to the spot combinations of A-4-F-1 and A-5-F-1 are schematically shown in Figs. 4(c) and 4(d), respectively, where the $c$-axis is aligned along $\mathrm{SrTiO}_{3}[100]$.

Furthermore, for the case of "B-3" at $\psi=-2.1^{\circ}$ in Fig. $3(\mathrm{~b})$, there are four corresponding spots at $\psi=87.9^{\circ}$ : D-1 and D-2 in Fig. 3(c), and "G-1" and "G-2" in Fig. 3(d). Here, combinations B-3-D-1 and B-3-D-2 have the $a$-axis along the $\mathrm{SrTiO}_{3}$ [100] direction while those of B-3-G-1 and B-3G-2 have $c$-axis along the same $\mathrm{SrTiO}_{3}[100]$ direction. It must be emphasized that the surface normal spots of these domains are the same as of B-3. This is schematically illustrated in Figs. 4(e)-4(h). It must be noted that based on the above results, two kinds of $a$-domains observed in Fig. 3(b) can be divided into three if we consider not only out-of-plane but also in-plane orientation. This shows that the number of domain types identified by the XRD measurements as shown in Fig. 3 is consistent with that determined by PFM measurements (Fig. 1).

There are (a2/a3) domain stripes in PFM as shown in Fig. 1. Based on the results shown in Figs. 3(c) and 3(d), the (a2/a3) or in-plane $90^{\circ}$ domain structure can be made by D-1-G-2 and D-2-G-1 combinations because the $\psi$ angle in Figs. 3(c) and $3(\mathrm{~d})$ is the same, $87.9^{\circ}$. In addition, the internal $\omega$ angle between D-1 and G-2 and D- 2 and G- 1 spots is $3.6^{\circ}$ as indicated in Fig. 5. This value is equal to that of the strain-free $\mathrm{PbTiO}_{3}$, which can be obtained as $2^{*} \arctan (c / a)-90^{\circ}$, where $a$ and $c$ are the lattice parameters of a strain-free state of $\mathrm{PbTiO}_{3}$. In fact, we found the inplane lattice parameters of $a$ - and c-axes $\left(a_{\|}\right.$and $\left.c_{\|}\right)$to be $a_{\|}=0.4147 \mathrm{~nm}$ and $c_{\|}=0.3896 \mathrm{~nm}$, which are in good agreement with the strain-free values.

For the next steps, we assign the XRD spots to the $c$ and a1-a3 domains identified in Figs. 1 and 2. As shown in Fig. 2(b), (c/a1) domain boundary is located along (011) plane. Based on the results discussed in the previous paper [see Fig. 5(b) of Ref 7], it can be concluded that the spot corresponding to $c$-domains as schematically shown in Fig. 3(a) is C-3 or $\mathrm{C}-4$. In addition, the (a2/c) domain boundary exists along (-101) plane [Fig. 2(c)], which shows a possibility of C-1 and C-4 based on the same discussion. Finally, C-4 corresponds to $c$-domain in Fig. 2 because it satisfies both requirements as mentioned above. Surface normals $a_{\perp}$ and $c_{\perp}$ are almost identical to the corresponding values found in strainfree $\mathrm{PbTiO}_{3}$ powder. This shows that A-2 and A-5 is a strainfree combination because its $\omega$ and $\psi$ axis interval between C-4 is almost $3.6^{\circ}$ along $\psi=0.7^{\circ}$ and $\omega=-0.7^{\circ}$ lines, respectively. This is almost identical to the strain-free $90^{\circ}$ domains. Therefore, "C-4-A-2" and C-4-A-5 are the combinations. On the other hand, similar alignment of (a2/a3) was found in both Figs. 2(d) and 5. This suggests that the spot B-3 in Fig. 3(b) of $\mathrm{PbTiO}_{3} 200$ along $\mathrm{SrTiO}_{3}$ [100] is likely corresponding to (a2/a3). Another possibility is B-1, whose tilting angle is also along $\psi=0^{\circ}$, but in the opposite direction. Thus, B-3 and B-1 are most likely the reflections of (a2/a3) in Fig. 2. Therefore, it is reasonable to assign the a1-domain connected with $c$-domain along $\mathrm{SrTiO}_{3}[010]$ to "A-2" rather than to A-5. In other words, C-4-A-2-B-3 or C-4-A-2-B-1 is more reasonable for the "c-a1-a2" combination than C-4-A-5-B-3 or C-4-A-5-B-1. Taking into account the fact that a thick film is expected to be more strained relaxed than a thin film, the "C-4-A-2-B-3" combination is more reasonable than C-4-A-2-B-1 since the angles of C-4 and B-3 are larger than those of C-4 and B-1 and also larger than the angles measured in thin films. ${ }^{14}$ As a result, the combination of C-4-A2-B-3 shown in Fig. 6(a) [the schematic projection of the $\omega-\psi$ maps overlapped by Figs. 3(a) and 3(b)] as red circles can be illustrated by the schematic in Fig. 6(b). Similarly, based on the same analogy, all spot combinations in Figs. 3(a) and 3(b) can be ascribed to the (a1/c) and (a2/a3) domain stripe structures shown in Fig. 6. Thus, it is shown experimentally that thick $\mathrm{PbTiO}_{3}$ films consist of the strainrelaxed (c/a1) and (a2/a3) domain configurations as was theoretically predicted by Roytburd. ${ }^{5}$ This suggests that strained-domain boundaries are localized between region I and region II, observed in stripe [see Fig. 1(a)] while others inside region I and region II are relaxed [(a2/a3) and (c1/ a1)]. This novel structure is different from the well-known type II structure because type II consists of the strained domain boundaries. ${ }^{9}$

At the present stage, we cannot perfectly understand how to contact the present type III structure with the substrates. Highly strained region is observed from the transmission electron microscopy observation at the interface between the film and the substrate, even if its thickness is very thin below $100 \mathrm{~nm}$. Tilting angle between $a$ and $c$ domains near the substrates was found to be smaller than that at the upper part of the films. These suggest the possibility of the existence of the strained structure between the type III structure (upper region having dominant volume fraction) and the substrate such as well-known structure type II. Detail analysis is under investigation.

In conclusion, complex domain structures consisting of one type of $c$-domains $\left(c_{1}\right)$ and three types of $a$-domains $\left(a_{1}\right.$, $a_{2}$, and $a_{3}$ ) have been visualized by PFM in $(100) /(001)$ - 
(a)
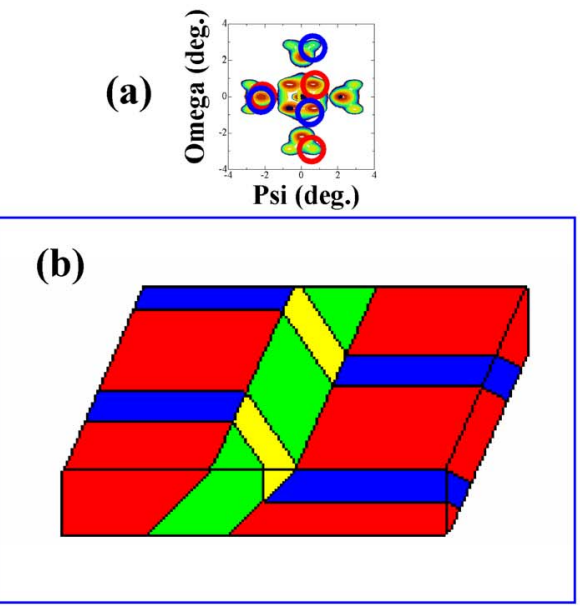

(c)

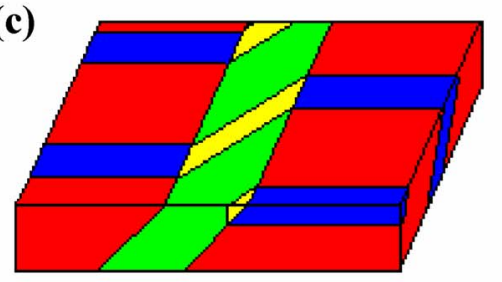

(d)

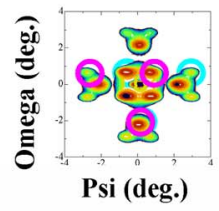

(e)

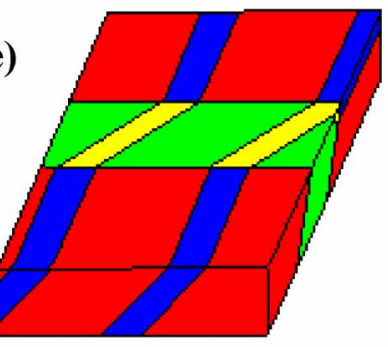

(f)

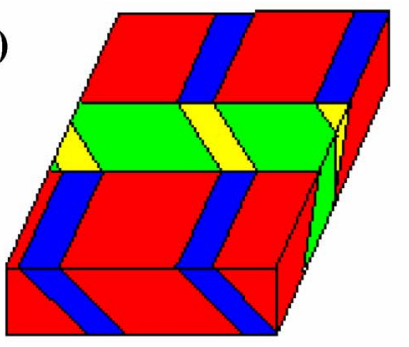

(g)
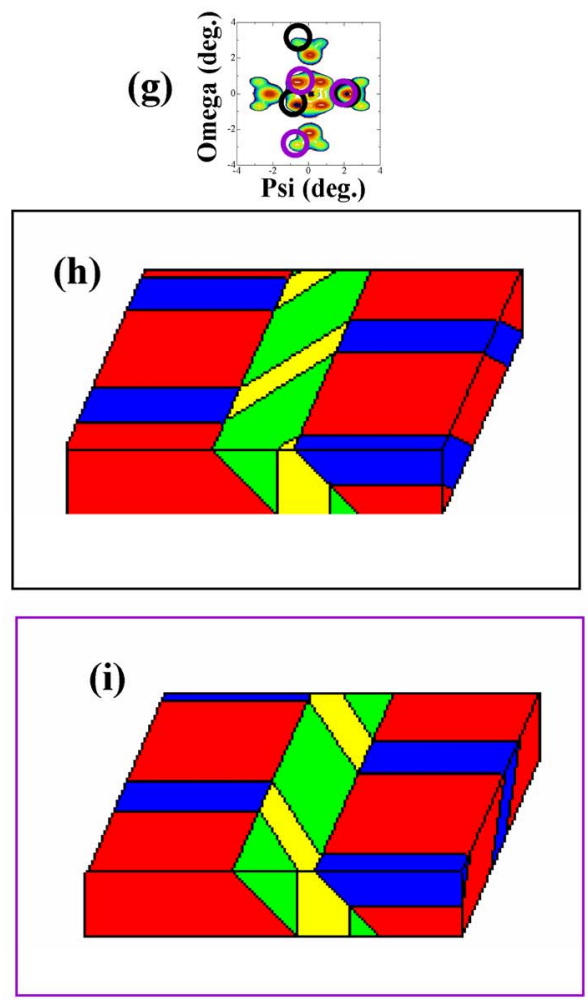

(j)

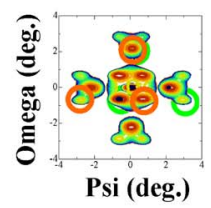

(k)

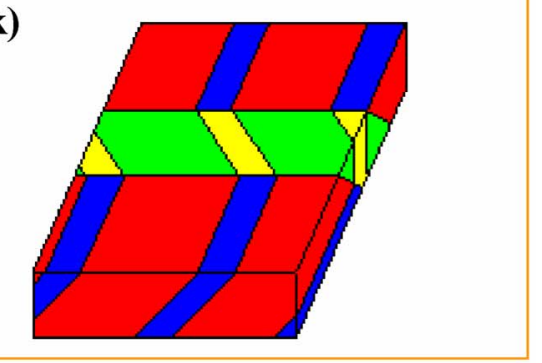

(I)

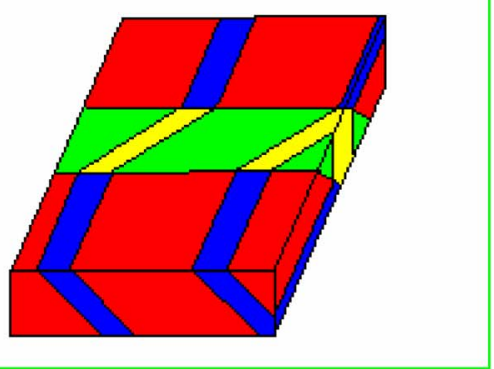

FIG. 6. (Color online) [(a), (d), (g), and (j)] Projections of $\omega-\psi$ scans of XRD patterns overlapped by Figs. 3(a) and 3(b). [(b), (c), (e), (f), (h), (i), (k), and (1)] schematic of the corresponding three-dimensional domain structures. Explanation is given in the text. oriented thick epitaxial $\mathrm{PbTiO}_{3}$ films grown on $\mathrm{Nb}$-doped (100) $\mathrm{SrTiO}_{3}$ substrates by pulsed MOCVD. Domain configurations consisting of (c/a1) and (a2/a3) stripes are found to be almost strain-free. These structures have been verified by XRD analysis and are in good agreement with the theoretically predicted domain patterns in thick films.

\section{ACKNOWLEDGMENTS}

Work at Argonne was supported by the U. S. Department of Energy, Office of Science, Office of Basic Energy Sciences, under Contract No. DE-AC02-06CH11357. 
${ }^{1}$ J. F. Scott and C. A. P. de Araujo, Science 246, 1400 (1998).

${ }^{2}$ M. Otsu, H. Funakubo, K. Shinnozaki, and N. Mizutani, Trans. Mater. Res. Soc. Jpn. 14B, 1655 (1994).

${ }^{3}$ Y. K. Kim, K. Lee, and S. Baik, J. Appl. Phys. 95, 236 (2004).

${ }^{4}$ J. S. Speck, A. C. Dykin, A. Seifert, A. E. Romanov, and W. Pompe, J. Appl. Phys. 78, 1696 (1995).

${ }^{5}$ A. L. Roytburd, Phys. Status Solidi A 37, 329 (1976)

${ }^{6}$ A. L. Roytburd and Y. Yu, Ferroelectrics 144, 137 (1993).

${ }^{7}$ H. Nakaki, Y. K. Kim, S. Yokoyama, R. Ikariyama, K. Nishida, K. Saito, and H. Funakubo, Appl. Phys. Lett. 91, 112904 (2007).

${ }^{8}$ A. Gruverman, A. Kholkin, A. Kingon, and H. Tokumoto, Appl. Phys. Lett. 78, 2751 (2001).

${ }^{9}$ C. S. Ganpule, V. Nagarajan, H. Li, A. S. Ogale, D. E. Steinhauer, S.
Agggarwal, E. Williams, R. Ramesh, and D. D. Wolf, Appl. Phys. Lett. 77, $292(2000)$

${ }^{10}$ K. Saito, T. Kurosawa, T. Akai, T. Oikawa, and H. Funakubo, J. Appl. Phys. 93, 545 (2003).

${ }^{11}$ C. M. Foster, Z. Li, M. Buckett, D. Miller, P. M. Baldo, L. E. Rehn, G. R. Bai, D. Guo, H. You, and K. L. Merkle, J. Appl. Phys. 78, 2607 (1995).

${ }^{12}$ K. Nagashima, M. Aratani, and H. Funakubo, Jpn. J. Appl. Phys., Part 2 39, L996 (2000).

${ }^{13}$ K. Nagashima and H. Funakubo, Jpn. J. Appl. Phys., Part 1 39, 212 (2000).

${ }^{14}$ S. V. Kalinin, B. J. Rodriguez, S. Jesse, J. Shin, A. P. Baddorf, P. Gupta, H. Jain, D. B. Williams, and A. Gruverman, Microsc. Microanal. 12, 1 (2006). 